\title{
Immunomodulatory Effect after Irreversible Electroporation in Patients with Locally Advanced Pancreatic Cancer
}

\author{
Chaobin He $\mathbb{D}^{1},{ }^{1}$ Jun Wang, ${ }^{1,2}$ Shuxin Sun, ${ }^{1}$ Yu Zhang, ${ }^{3}$ and Shengping Li ${ }^{1}{ }^{1}$ \\ ${ }^{1}$ Department of Hepatobiliary and Pancreatic Surgery, State Key Laboratory of Oncology in South China, \\ Collaborative Innovation Center for Cancer Medicine, Sun Yat-sen University Cancer Center, Guangzhou 510060, China \\ ${ }^{2}$ Department of Ultrasonics, State Key Laboratory of Oncology in South China, Collaborative Innovation Center for Cancer Medicine, \\ Sun Yat-sen University Cancer Center, Guangzhou 510060, China \\ ${ }^{3}$ State Key Laboratory of Ophthalmology, Zhongshan Ophthalmic Center, Sun Yat-sen University, Guangzhou, \\ Guangdong 510060, China
}

Correspondence should be addressed to Shengping Li; lishengp@mail.sysu.edu.cn

Received 15 March 2019; Revised 18 April 2019; Accepted 23 April 2019; Published 12 May 2019

Guest Editor: Nathaniel Weygant

Copyright (C) 2019 Chaobin He et al. This is an open access article distributed under the Creative Commons Attribution License, which permits unrestricted use, distribution, and reproduction in any medium, provided the original work is properly cited.

\begin{abstract}
Purpose. Irreversible electroporation (IRE) has been demonstrated to be a safe and effective method for locally advanced pancreatic cancer (LAPC). The aim of this study was to evaluate the immunomodulatory effect after IRE and to evaluate the prognostic value of variations of the immune parameters in LAPC patients after IRE. Methods. Peripheral blood samples of 34 patients were obtained preoperatively and on the third day (D3) and seventh day (D7) after IRE, respectively. The phenotypes of lymphocytes were analyzed by flow cytometry, and dynamic changes of serum levels of cytokines, complement, and immunoglobulin were assayed by enzyme-linked immunosorbent assay. Receiver operating characteristic (ROC) curve and concordance index (C-index) were used to compare the survival predictive ability. Results. There was a transitory decrease followed by a steady increase for CD $4^{+} \mathrm{T}$ cell, $\mathrm{CD}^{+}$T cell, NK cell, IL-2, C3, C4, and IgG while a reverse trend was detected for Treg cell, IL-6, and IL10 after IRE. The alteration of $\mathrm{CD}^{+} \mathrm{T}$ cell between $\mathrm{D} 3$ and $\mathrm{D} 7$ was identified as a prognostic factor for both overall survival (OS) and progression-free survival (PFS). The values of ROC curve (AUC) and C-indexes of the alteration of CD8 ${ }^{+} \mathrm{T}$ cell for OS and PFS were 0.816 and 0.773 and 0.816 and 0.639 , respectively, which were larger than those of other immune or inflammation-based indexes. Conclusions. This study presented the first evidence of IRE-based immunomodulatory in patients with LAPC. The alteration of CD8 ${ }^{+} \mathrm{T}$ cell between $\mathrm{D} 3$ and D7 showed relatively good performance and could be used as an effective tool for prognostic evaluation for LAPC patients after IRE.
\end{abstract}

\section{Introduction}

Pancreatic adenocarcinoma is a lethal disease with extremely poor prognosis, which also represented the seventh and sixth leading causes of cancer-related death in the world and in China, respectively. The 5 -year survival rate is only $5 \%[1,2]$. Surgical resection is the only chance to obtain curative treatment while it is only suitable for less than $20 \%$ of patients with this disease [3]. Approximately $40 \%$ of new cases are diagnosed with locally advanced pancreatic cancer (LAPC), which is characterized by the involvement of major vascular structures, such as celiac trunk, superior mesenteric artery, leading to unresectable but nonmetastatic diseases [4]. Currently, the treatment for LAPC remains a huge challenge due to the poor prognoses of this disease. Limited responses and little impact on survival or life were achieved after the standard treatments, which was mainly systemic chemotherapy $[5,6]$. Moreover, the high rates of adverse events due to the toxicity of chemotherapy limited the use and promotion of treatment, such as the combination chemotherapy of 5-fluorouracil, leucovorin, irinotecan, and oxaliplatin (FOLFIRINOX), even though it was shown to display some progression in improving the survival of patients with LAPC [7-9]. Therefore, it is necessary to evaluate new treatment to optimize common therapeutic approaches. Nowadays, local therapies were shown to improve the prognosis of LAPC patients with varying degrees of success [10]. 
Irreversible electroporation (IRE), a nonthermal ablation technique, is established as a local ablative therapy for patients with LAPC with promising outcomes of increasing overall survival (OS) from 12 months to 25 months [11]. It is a novel local destructive method based on the transmission of high voltage currents through the tumor via needles, leading to cell membrane defects and apoptotic death $[12,13]$. Additionally, during the induction process of apoptotic death by IRE, the structure and composition of the tumor microenvironment are changed, inducing an intense inflammatory cell response, which is characterized by the infiltration of immune cells [14]. It was shown that this IRE-induced immunomodulatory was not only limited to the ablated areas, but also a systemic reaction [15]. Thus, IRE could be regarded as a potential immunomodulatory treatment and might induce extensive changes of immune cells or indexes after ablation.

So far, data is rare on the predictive factors of IRE outcome in patients with LAPC. For this novel and powerful treatment of LAPC, further prognostic markers are urgently needed to choose patients with relatively better prognosis. Moreover, early information of the efficacy of treatment during the first days after IRE would be highly appreciated as therapy may be intensified by other treatments, such as immune therapy, chemotherapy, and radiotherapy, while the regular evaluation of therapy by imaging is only done about 1 month after IRE treatment. For the candidates of predictive factors, circulating biochemical markers may be the promising ones, for their relationship with cancer disease, the immediate therapy effect, and the immunological response of the organism to treatment. More importantly, as failures were achieved for the immune-checkpoint therapies in pancreatic cancer due to the low rates of neoantigen expression and mutation events [16], exploring the alterations and evaluating the prognostic effect of immune cells and indexes might open the prospect of using immune-checkpoint therapies in patients with LAPC.

Here, immunomodulatory effect of IRE was examined by analyzing alterations of several immune cells and indexes in patients with LAPC. We aimed to evaluate the response to IRE therapy during the early treatment phase and identify their role in prognosis.

\section{Materials and Methods}

2.1. Patients. This study was retrospectively designed. Consecutive patients who were newly diagnosed with LAPC at Sun Yat-sen University Cancer Center between August 2015 and August 2017 were included in this study. The inclusion criteria were as follows: (1) pathologically confirmed pancreatic adenocarcinoma and radiologically confirmed LAPC. LAPC was defined per the seventh edition of the AJCC staging system for pancreatic cancer, which describes LAPC as arterial encasement of either the celiac axis or superior mesenteric artery or unreconstructable superior mesenteric or portal vein involvement, with no evidence of metastatic disease from abdominal and thoracic computed tomography $[17,18]$; (2) IRE therapy as the initial treatment. A total of 11 patients were excluded based on the following exclusion criteria: (1) other treatments, including surgical resection and RFA before IRE (seven patients); (2) existing metastatic implants before IRE (one patient); (3) heart arrhythmia and a history of second primary malignant tumors (one patient); (4) missing information of parameters or lost to follow-up (two patients). This study was approved by the Institutional Review Board of Sun Yat-sen University Cancer Center. All procedures performed in present study involving human participants were in accordance with the ethical standards of institutional and/or national research committees and the 1964 Helsinki Declaration and its later amendments or similar ethical standards. Written informed consent was obtained from patients prior to treatment.

2.2. Clinical Data Collection. The following clinical and radiological data were retrieved from medical record archived at Sun Yat-sen University Cancer Center, including age, gender, tumor size, tumor grade, tumor site, white blood cell (WBC) count, platelet (PLT) count, serum levels of alanine transaminase (ALT), aspartate aminotransferase (AST), alkaline phosphatase (ALP), glutamyl transpeptidase (GGT), albumin (ALB), total bilirubin (TBIL), indirect bilirubin (IBIL), Creactive protein (CRP), carcinoembryonic antigen (CEA), and carbohydrate antigen 19-9 (CA19-9). The inflammationbased indexes, including neutrophil-to-lymphocyte ratio (NLR), platelet-to-lymphocyte ratio (PLR), prognostic index (PI), and modified Glasgow Prognostic Score (mGPS), were also entered into this study. The thresholds for the clinical or radiological variables were used as the cutoff values. With the cutoff value of 1.47 and 165.29, NLR and PLR were associated with the optimal Youden indexes for OS and progression-free survival (PFS) prediction, respectively. The defined score of other inflammation-based indexes, such as PI and mGPS, had been described in previous studies [19].

2.3. Treatment Procedure. The NanoKnife IRE equipment from Angiodynamics System (Queensbury, NY, USA) was used. General anesthesia with deep neuromuscular block was adopted. To create an electric field around the tumor, 3 to 6 probes were used according to the size and location of the tumor. Ultrasound was used to guide the placement of all probes, and adequate space between probes was then confirmed. The generator unit software was used to analyze the probe configuration data of the ultrasound and provided optimal voltage and pulse length delivery. If the tumor size was larger than $1.5 \mathrm{~cm}$ in the axial plane, a pull-back technique with the same procedure was performed to cover the entire area of ablation.

2.4. Sample Collection. All blood samples were collected before the hypothesis of this study was known. The blood samples were collected using $\mathrm{Na}$-heparin plasma tubes from enrolled patients before IRE (preOP) and then on days 3 (D3) and 7 (D7) after IRE. Isolation of peripheral blood mononuclear cells (PBMCs) was processed immediately using Hypaque-Ficoll (Promega) and frozen in liquid nitrogen in $5 \%(\mathrm{v} / \mathrm{v})$ plus $95 \%(\mathrm{v} / \mathrm{v})$ autologous serum [20]. 
2.5. Flow Cytometry Analysis. Frozen PBMCs were thawed in a $37^{\circ} \mathrm{C}$ water bath and then cultured overnight at $37^{\circ} \mathrm{C}$ in RPMI-1640 (Gibco BRL) supplemented with 5\% human $\mathrm{AB}$ type serum and labeled with FITC-, APC-, and/or PEconjugated murine anti-human monoclonal antibodies. The CD3, CD3CD4, CD3CD8, CD3CD16CD56, and CD4CD25 phenotype of lymphocytes were sequentially analyzed by flow cytometry (FACS caliber, 4 color system, BD Bioscience, CA, US).

2.6. Assays of Immune Parameters. The quantitative sandwich enzyme immunoassay technique (ELISA kit, R\&D system, Minneapolis, MN) was adopted to measure serum concentrations of cytokines, including IL-2, IL6, IL-10, interferon$\gamma(\mathrm{IFN}-\gamma)$, and tumor-necrosis factor (TNF). During the procedure of measure, 50 to $100 \mu \mathrm{l}$ of assay diluent was added to the 96-well polystyrene microplate, which was precoated with murine monoclonal antibody against IL-2, IL-6, IL-10, IFN- $\gamma$, and TNF. Serum samples were incubated at $37^{\circ} \mathrm{C}$ for 2 hours and then the plates were aspired and washed three times. Same incubation was repeated after 200 micoliters of conjugate was added. Then, plates were incubated at $37^{\circ} \mathrm{C}$ for 20 to 30 minutes after $200 \mu \mathrm{l}$ of substrate solution was added. Finally, $50 \mu \mathrm{l}$ of stop solution was added to the plates. A microplate reader (ClinicalBio 128c, Austria) was used to read optimal density (OD) within 30 minutes at $450 \mathrm{~nm}$ wavelength, whose references were set to 550 and $620 \mathrm{~nm}$.

A Beckman ARRAY 360 System (Beckman Coulter, Galway, Ireland) was used to evaluate the concentrations of several humoral immune parameters, including C3, C4, IgA, IgM, and IgG. Specific antibodies were measured by enzymelinked immunosorbent assay (ELISA). After the incubation, the microplate reader (ClinicalBio 128c, Austria) was used to read OD within 30 minutes at $450 \mathrm{~nm}$ wavelength, whose reference was set to $630 \mathrm{~nm}$ [20].

2.7. Follow-Up. The follow-up procedure was performed in accordance with previous publications and recommendations [21, 22]. OS was defined as the duration from treatment until death or the last follow-up. PFS was defined as the duration from treatment until the date when disease progression was diagnosed or until the last follow-up. The last follow-up was completed on September 30, 2018.

2.8. Statistical Analysis. Continuous variables were compared using an independent sample t-test and the Mann-Whitney $\mathrm{U}$ test. Binary categorical variables were compared using the chi-square test. OS and PFS curves were analyzed using the Kaplan-Meier method, and differences between the groups were identified using the log-rank test. Univariate analysis was performed to assess the significance of parameters. Multivariate analysis was performed using the Cox regression model for the variables that were found to be significant in the univariate analysis, and the corresponding $95 \%$ confidence intervals (CIs) were calculated. ROC curves and C-indexes were used to compare the survival predictive ability. Twotailed $\mathrm{P}$ values $<0.05$ were considered statistically significant. All statistical analyses were performed using the R statistical package ( $\mathrm{R}$ software version 3.4.2; R Foundation for Statistical Computing, Vienna, Austria).

\section{Results}

3.1. Patient Characteristics. In the present study, a total of 34 patients with LAPC were retrospectively included in this study. All patients have received IRE therapy. There were 18 (52.9\%) female patients and $16(47.1 \%)$ male patients. The median age was 59.5 years (range $45-73$ years). Patient characteristics were summarized in Table 1 . Large size and moderate differentiation were the most commonly seen features of tumors. Most patients had lower values of inflammatory indexes, such as PLR, PI, and mGPS, while patients with higher values of NLR occupied the majority of all patients. For the whole study cohort, there were only 4 patients whose TBIL was higher than $100 \mathrm{umol} / \mathrm{L}$. Complications after IRE treatment in patients with LAPC were also evaluated (Table 2). The most frequently reported complications were pain ( 3 of 34 patients) and hypotension ( 3 of 34 patients).

3.2. Modulation of Circulating Immune Cells. To investigate how IRE influences circulating immune cells, these cells were phenotypically characterized by evaluating the absolute number of helper $\mathrm{T}$ cell $\left(\mathrm{CD} 4^{+} \mathrm{T}\right.$ cell, identified as $\left.\mathrm{CD} 3^{+} \mathrm{CD} 4^{+}\right)$, cytotoxic T cell $\left(\mathrm{CD}^{+}{ }^{\mathrm{T}}\right.$ cell, identified as $\left.\mathrm{CD}^{+}{ }^{+} \mathrm{CD} 8^{+}\right)$, regulatory $\mathrm{T}$ cell (Treg, identified as $\mathrm{CD} 4^{+} \mathrm{CD} 25^{+} \mathrm{FoxP} 3^{+}$), and natural killer cell (NK cell, identified as $\mathrm{CD}^{-} \mathrm{CD}^{+} 6^{+} \mathrm{CD} 56^{+}$) before (preOP) and after IRE treatment (D3 and D7). It was shown that the absolute numbers of $\mathrm{CD}^{+} \mathrm{T}$ cell $(p<0.05)$, $\mathrm{CD}^{+} \mathrm{T}$ cell $(p<0.05)$, and NK cell $(p<0.01)$ were decreased immediately after IRE (D3), followed by a steady increase in the next few days (D7) $(p<0.001)$. However, the trend for Treg cell reversed between preOP and D7 $(p<0.05)$. The NK cell showed the most dramatic inverse effect for each time interval. Huge alterations of $\mathrm{CD} 4^{+} \mathrm{T}$ cell and $\mathrm{CD} 8^{+} \mathrm{T}$ cell were observed while there was a significant decrease in the ratio of $\mathrm{CD} 4^{+} \mathrm{T}$ cell to $\mathrm{CD} 8^{+} \mathrm{T}$ cell from D3 to $\mathrm{D} 7(p<0.05)$ (Figure 1).

\subsection{Modulation of Circulating Cytokines and Humoral} Immune Parameters. For a more complete understanding of the IRE-associated alteration of immune, analyses of the plasma concentration of several cytokines were conducted. Marked changes were observed for interleukin-2 (IL-2) $(p<0.05)$, IL-6 $(p<0.001)$, and IL-10 ( $p<0.01)$. IRE dramatically increased circulating IL- 6 and IL-10 at D3 but these decreased at D7 (all $p<0.05$ ). Although no changes of IL-2 at D3 were observed, there was a significant increase from D3 to D7 $(p<0.05)$. On the contrary, IRE did not significantly alter plasma concentration of IFN- $\gamma$ and TNF $(p>0.05)$. Moreover, we analyzed the plasma concentration of several general humoral immune parameters (complement: C3 and C4; immunoglobulin: $\operatorname{IgA}, \operatorname{IgG}$, and $\operatorname{IgM})$. C3, C4, and $\operatorname{IgG}$ notably decreased immediately after IRE (D3) (all $p<0.05)$ but significantly increased within one week (all $p<0.01)$. There were no significant changes for concentration of $\operatorname{IgA}$ and $\operatorname{IgM}$ (all $p>0.05$ ) (Figure 2). 
TABLE 1: Characteristics of patients with LAPC undergoing IRE therapy.

\begin{tabular}{|c|c|c|c|}
\hline Characteristics & & Number & Percentage (\%) \\
\hline Patients & & 34 & 100 \\
\hline \multirow{2}{*}{ Age (years) } & $\leq 60$ & 19 & 55.9 \\
\hline & $>60$ & 15 & 44.1 \\
\hline \multirow{2}{*}{ Gender } & Female & 18 & 52.9 \\
\hline & Male & 16 & 47.1 \\
\hline \multirow{3}{*}{ Tumor size $(\mathrm{cm})$} & $\leq 2$ & 1 & 2.9 \\
\hline & $2 \sim 4$ & 19 & 55.9 \\
\hline & $>4$ & 14 & 41.2 \\
\hline \multirow{3}{*}{ Tumor grade } & Well & 2 & 5.9 \\
\hline & Moderate & 19 & 55.9 \\
\hline & Poor & 13 & 38.2 \\
\hline \multirow{2}{*}{ Tumor site } & Head & 17 & 50.0 \\
\hline & Body / Tail & 17 & 50.0 \\
\hline \multirow{2}{*}{ WBC $(* 109)$} & $\leq 10$ & 30 & 88.2 \\
\hline & $>10$ & 4 & 11.8 \\
\hline \multirow{2}{*}{ HGB (g/L) } & $\leq 120$ & 10 & 29.4 \\
\hline & $>120$ & 24 & 70.6 \\
\hline \multirow{2}{*}{ PLT $(* 109)$} & $\leq 300$ & 29 & 85.3 \\
\hline & $>300$ & 5 & 14.7 \\
\hline \multirow{2}{*}{$\operatorname{ALT}(\mathrm{U} / \mathrm{L})$} & $\leq 40$ & 25 & 73.5 \\
\hline & $>40$ & 9 & 26.5 \\
\hline \multirow{2}{*}{ AST (U/L) } & $\leq 40$ & 28 & 82.4 \\
\hline & $>40$ & 6 & 17.6 \\
\hline \multirow{2}{*}{$\operatorname{ALP}(\mathrm{U} / \mathrm{L})$} & $\leq 100$ & 18 & 52.9 \\
\hline & $>100$ & 16 & 47.1 \\
\hline \multirow{2}{*}{ GGT (U/L) } & $\leq 45$ & 18 & 52.9 \\
\hline & $>45$ & 16 & 47.1 \\
\hline \multirow{2}{*}{$\operatorname{ALB}(g / L)$} & $\leq 40$ & 3 & 8.8 \\
\hline & $>40$ & 31 & 91.2 \\
\hline \multirow{2}{*}{ TBIL (umol/L) } & $\leq 20.5$ & 26 & 76.5 \\
\hline & $>20.5$ & 8 & 23.5 \\
\hline \multirow{2}{*}{ IBIL (umol/L) } & $\leq 15$ & 30 & 88.2 \\
\hline & $>15$ & 4 & 11.8 \\
\hline \multirow{2}{*}{ CRP (ng/L) } & $\leq 3$ & 25 & 73.5 \\
\hline & $>3$ & 9 & 26.5 \\
\hline \multirow{2}{*}{ CEA (ng/mL) } & $\leq 5$ & 20 & 58.8 \\
\hline & $>5$ & 14 & 41.2 \\
\hline \multirow{2}{*}{ CA19-9 (U/ml) } & $\leq 35$ & 8 & 23.5 \\
\hline & $>35$ & 26 & 76.5 \\
\hline \multirow{2}{*}{ NLR } & $\leq 1.47$ & 9 & 26.5 \\
\hline & $>1.47$ & 25 & 73.5 \\
\hline \multirow{2}{*}{ PLR } & $\leq 165.29$ & 22 & 64.7 \\
\hline & $>165.29$ & 12 & 35.3 \\
\hline \multirow{2}{*}{ PI } & 0 & 25 & 73.5 \\
\hline & 1 & 9 & 26.5 \\
\hline \multirow{3}{*}{ mGPS } & 0 & 29 & 85.3 \\
\hline & 1 & 4 & 11.8 \\
\hline & 2 & 1 & 2.9 \\
\hline
\end{tabular}

LAPC, locally advanced pancreatic cancer; IRE, irreversible electroporation; WBC, white blood cell count; PLT, platelet count; ALT, alanine transaminase; AST, aspartate aminotransferase; ALP, alkaline phosphatase; GGT, glutamyl transpeptidase; ALB, albumin; TBIL, total bilirubin; IBIL, indirect bilirubin; CRP, C-reactive protein; CEA, carcinoembryonic antigen; CA19-9, carbohydrate antigen 19-9; NLR, neutrophil-to-lymphocyte ratio; PLR, platelet-to-lymphocyte ratio; PI, prognostic index; mGPS, modified Glasgow Prognostic Score. 


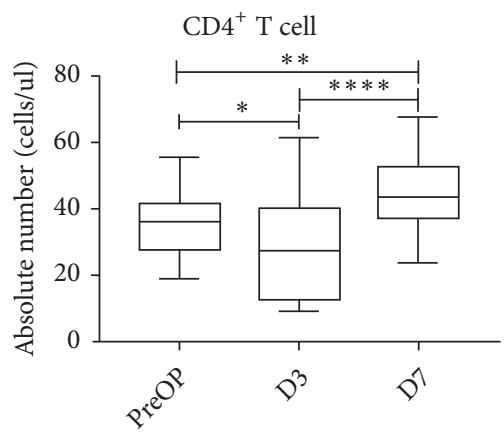

(a)

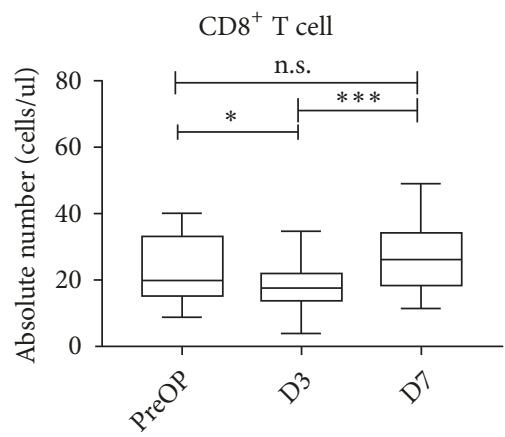

(b)

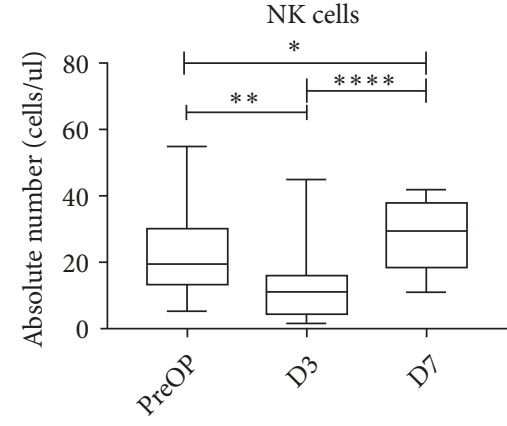

(c)

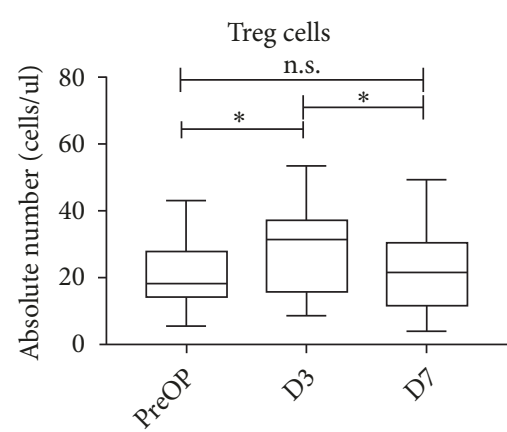

(d) (e)

FIGURE 1: Distribution of serum concentration of CD4+ T cell (a), CD 8+ T cell (b), NK cell (c), Treg cell (d), and the ratio of CD4+ T cell/CD8+ $\mathrm{T}$ cell (e) before, 3 days, and 7 days after IRE therapy, indicating medians, interquartile range, 5th and 95th percentiles, and extreme values. ${ }^{*} \mathrm{p}<0.05 ;{ }^{* *} \mathrm{p}<0.01 ;{ }^{* * *} \mathrm{p}<0.001 ;{ }^{* * * *} \mathrm{p}<0.0001$. NK cell: natural kill cell; Treg cell: regulatory T cell; IRE: irreversible electroporation.

TABLE 2: Complications after IRE treatment in patients with LAPC.

\begin{tabular}{lc}
\hline Complications & Number \\
\hline Hypotension & 3 \\
Hypokalemia & 2 \\
Fatigue & 2 \\
Vomiting & 1 \\
Diarrhea & 2 \\
Thrombosis & 2 \\
Ascites & 1 \\
Pain & 3 \\
Muscle weakness & 1 \\
\hline
\end{tabular}

Abbreviations as in Table 1

3.4. Comparison of Survival Stratified by Changes of Immune Cells and Parameters. In the whole study cohort, there were $27(79.4 \%)$ patients alive at the end of follow-up. The cumulative 1-year and 2-year OS rates were $69.9 \%$ and $52.4 \%$, respectively. To evaluate the prognostic value of immune cells and parameters, the elevated or decreased group of these variables was defined by the threshold, which was the median value of the alterations between D3 and D7. In the subgroup analyses for OS, patients with an increase of $\mathrm{CD} 4^{+} \mathrm{T}$ cell $(p=0.047), \mathrm{CD}^{+} \mathrm{T}$ cell $(p<0.001)$, and NK cell
( $p=0.013)$ or a decrease of Treg cell $(p=0.015)$ had significant better OS than others. There were no significant differences with regard to OS when it was stratified by changes of cytokines, including IL-2, IL-6, and IL-10 (all $p>0.05$ ). In addition to these variables, alteration of $\mathrm{C} 3, \mathrm{C} 4$, and $\operatorname{IgG}$ did not lead to significant differences in OS (all $p>0.05$ ) (Figure 3). Regarding PFS, significant survival benefit could be obtained from an increase of $\mathrm{CD}^{+} \mathrm{T}$ cell $(p=0.048)$ while the alterations of other immune cells or parameters were not significantly associated with PFS (Figure 4).

3.5. Univariate and Multivariate Analyses of OS and PFS. In Cox regression analysis, the increase of $\mathrm{CD}^{+} \mathrm{T}$ cell was associated with increased OS and PFS [Elevated vs Nonelevated, OS, HR=0.039, 95\%CI, 0.002-0.780, $p=0.034$; PFS, HR $=0.418,95 \% \mathrm{CI}, 0.138-0.954, p=0.049]$ in all patients. Moreover, there were no other prognostic factors for OS and the remaining one prognostic factor for PFS was NLR $(>1.47$ vs $\leq 1.47, \mathrm{HR}=3.425,95 \% \mathrm{CI}, 1.002-12.616, p=0.046)$ (Table 3).

3.6. Comparison of Predictive Value of the Immune Cells and Inflammation-Based Indexes. ROC curves were used to compare the sensitivity and specificity of survival prediction among the immune cells, parameters and inflammationbased indexes (Figure 5). The values of AUC of alteration 


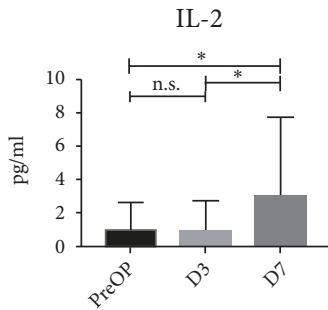

(a)

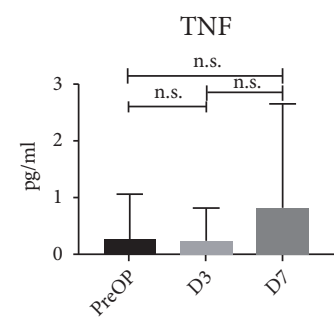

(e)

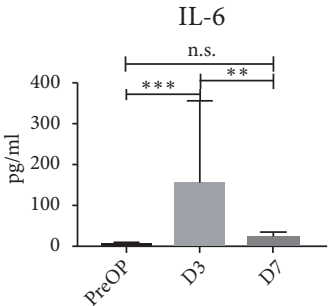

(b)

C3

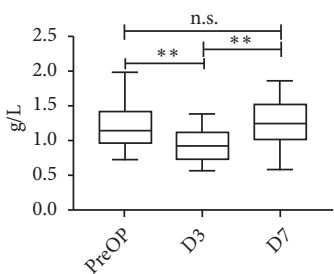

(f)

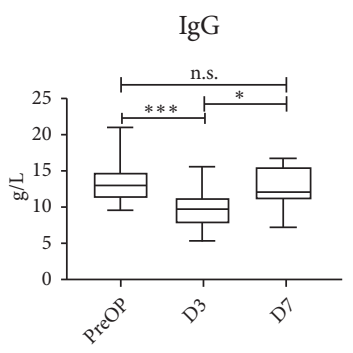

(i)

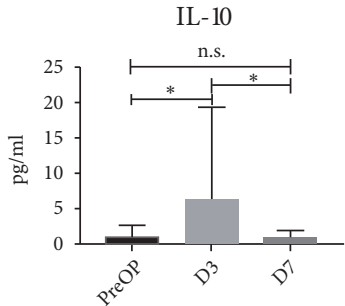

(c)

C4

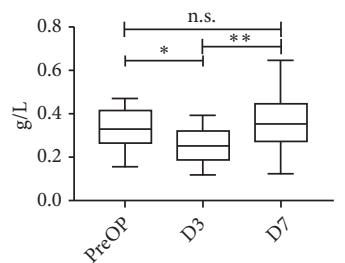

(g)

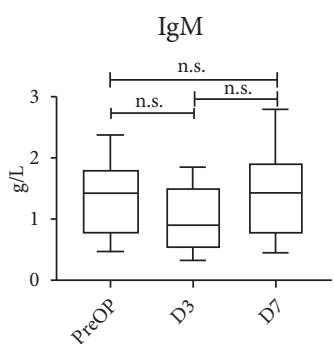

(j)

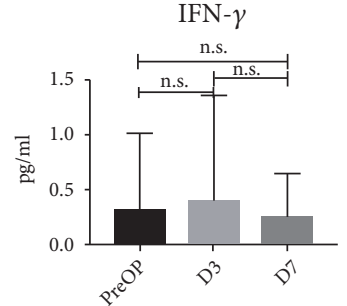

(d)

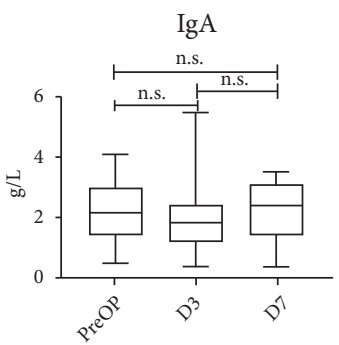

(h)

Figure 2: Distribution of serum concentration of IL-2 (a), IL-6 (b), IL-10 (c), IFN- $\gamma$ (d), TNF (e), C3 (f), C4 (g), IgA (h), IgG (i), IgM (j) before, 3 days, and 7 days after IRE therapy. ${ }^{*} \mathrm{p}<0.05{ }^{* *} \mathrm{p}<0.01 ;{ }^{* * *} \mathrm{p}<0.001$. IL: interleukin; IFN- $\gamma$ : interferon- $\gamma$; TNF: tumor-necrosis factor; C3: complement 3; C4: complement 4; IgA: immunoglobulin A; IgG: immunoglobulin G; IgM: immunoglobulin M; IRE: irreversible electroporation.

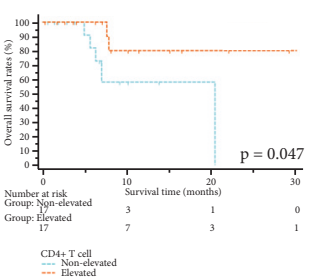

(a)

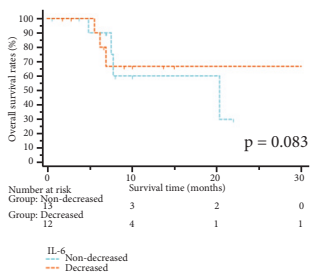

(f)

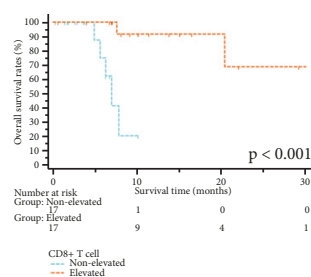

(b)

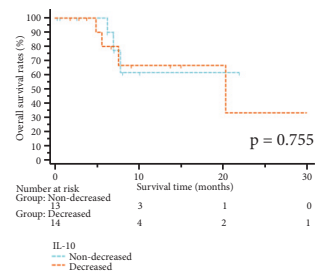

(g)

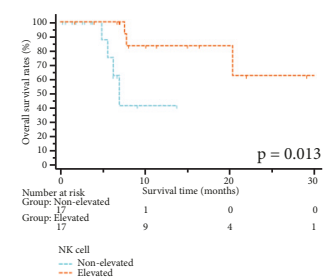

(c)

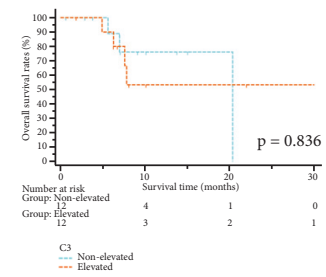

(h)

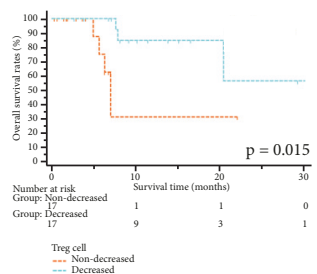

(d)

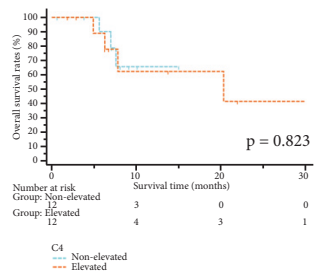

(i)

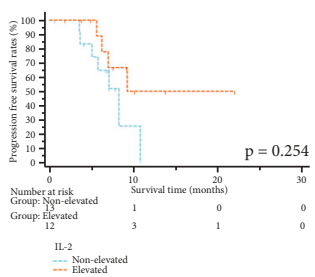

(e)

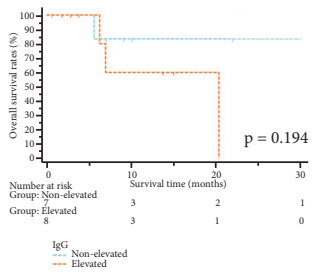

(j)

Figure 3: The survival curves of overall survival stratified by immune cells and parameters. Alteration of CD4+ T cell (a), CD 8+ T cell (b), NK cell (c), Treg cell (d), IL-2 (e), IL-6 (f), IL-10 (g), C3 (h), C4 (i), and IgG (j). NK cell: natural kill cell; Treg cell: regulatory T cell; IL: interleukin; C3: complement 3; C4: complement 4; IgG: immunoglobulin G; IRE: irreversible electroporation. 


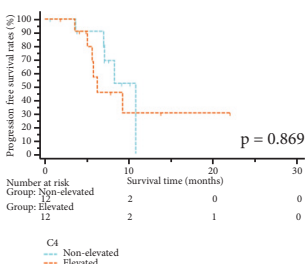

(a)

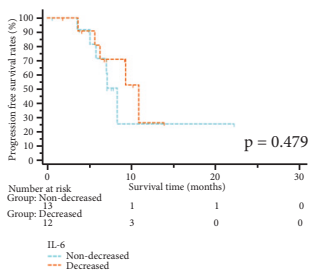

(f)

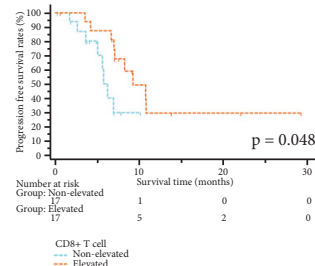

(b)

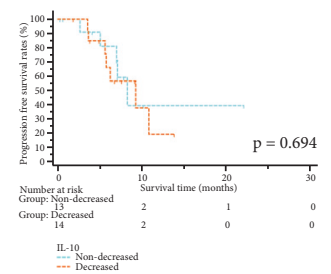

(g)

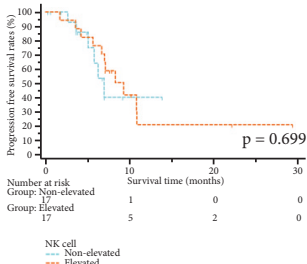

(c)

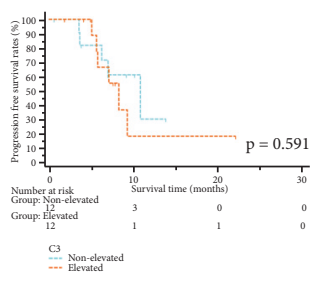

(h)

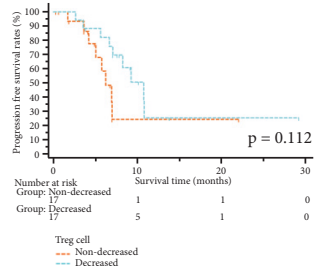

(d)

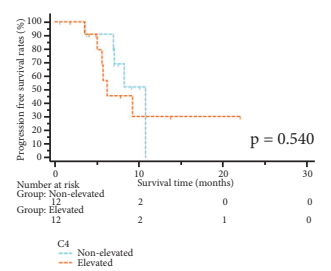

(i)

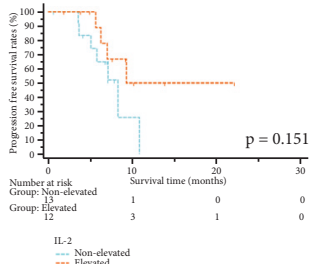

(e)

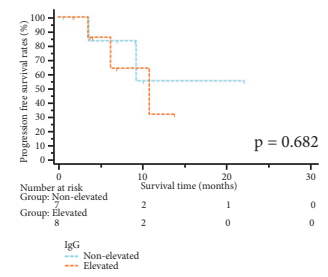

(j)

Figure 4: The survival curves of progression-free survival stratified by immune cells and parameters. Alteration of CD4+ T cell (a), CD 8+ T cell (b), NK cell (c), Treg cell (d), IL-2 (e), IL-6 (f), IL-10 (g), C3 (h), C4 (i), and IgG (j). NK cell: natural kill cell; Treg cell: regulatory T cell; IL: interleukin; C3: complement 3; C4: complement 4; IgG: immunoglobulin G; IRE: irreversible electroporation.

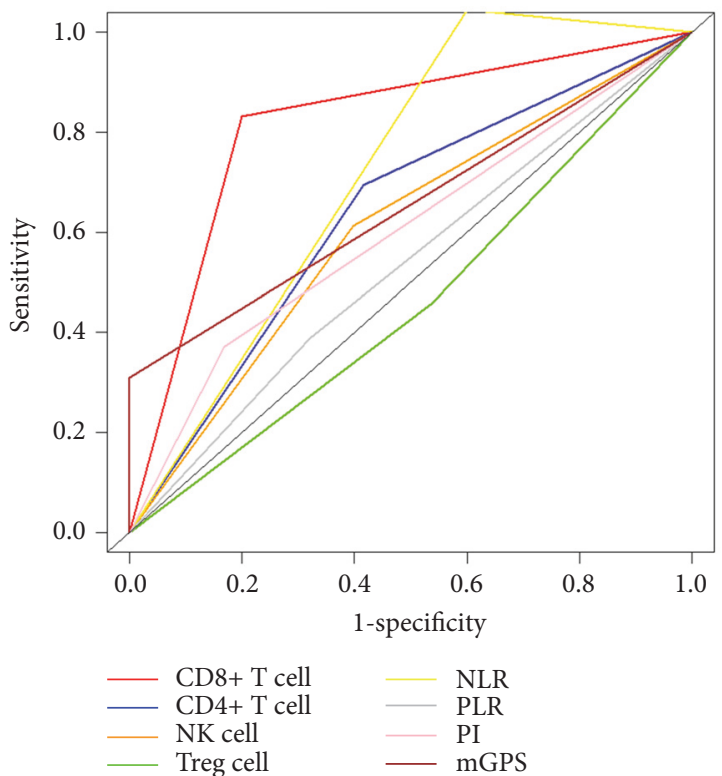

(a)

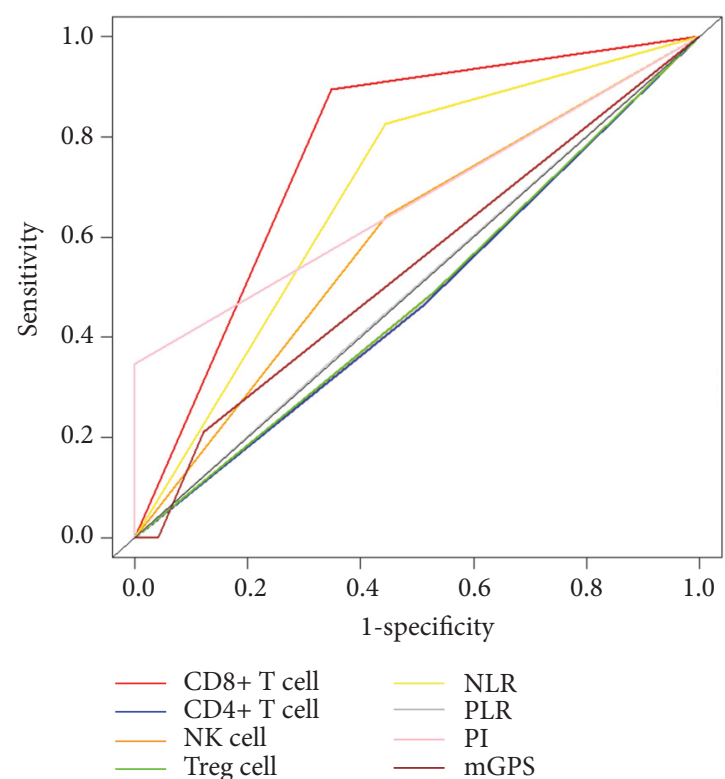

(b)

FIGURE 5: Comparison of ROC curves of alteration of immune cells, cytokines, or inflammation-based indexes, for predicting OS (a) and PFS (b) in patients with LAPC after IRE therapy. ROC: receiver operating characteristic; OS: overall survival; PFS: progression-free survival; LAPC: locally advanced pancreatic cancer; IRE: irreversible electroporation.

of $\mathrm{CD}^{+} \mathrm{T}$ cell for OS and PFS prediction were 0.816 and 0.773 , respectively, which were both higher than those of other immune parameters or inflammation-based indexes (Table 4). In terms of comparisons of C-indexes for OS prediction, the value of alteration of $\mathrm{CD}^{+} \mathrm{T}$ cell was 0.816 (95\%CI 0.711-0.921), which was higher than that of other factors. In terms of PFS prediction, $\mathrm{CD}^{+} \mathrm{T}$ cell also displayed relatively high value of 0.639 (95\%CI $0.523-0.755)$, showing significant better predictive power (Table 5 ).

\section{Discussion}

In this study, an immunomodulatory effect was demonstrated by altering lymphocytes, cytokines, and humoral immune parameters in patients with LAPC after IRE. It was the first evidence for IRE-based immune modulation in LAPC patients. It was shown that there was a transitory decrease followed by a steady increase for $\mathrm{CD} 4^{+} \mathrm{T}$ cell, $\mathrm{CD} 8^{+} \mathrm{T}$ cell, NK cell, IL-2, C3, C4, and IgG while a reverse trend was observed 


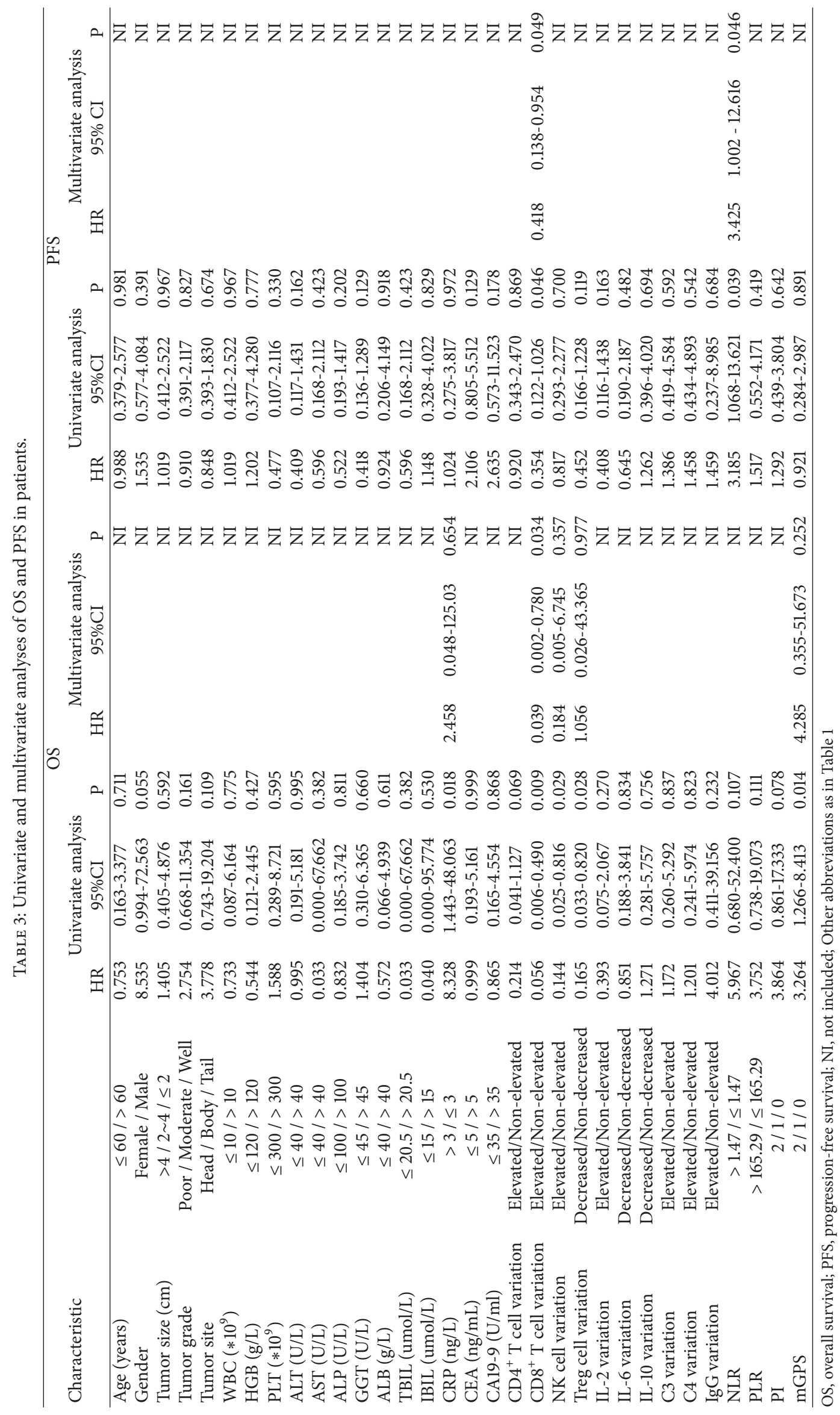




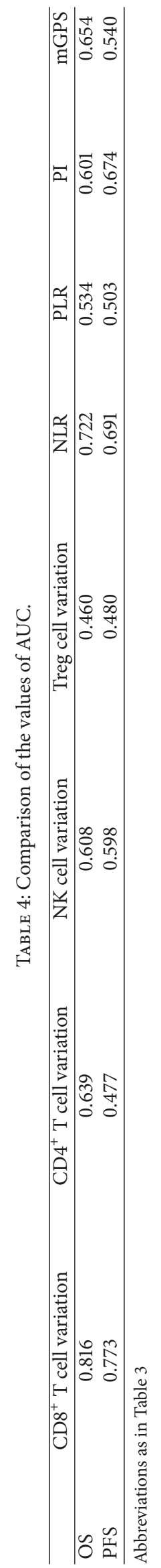




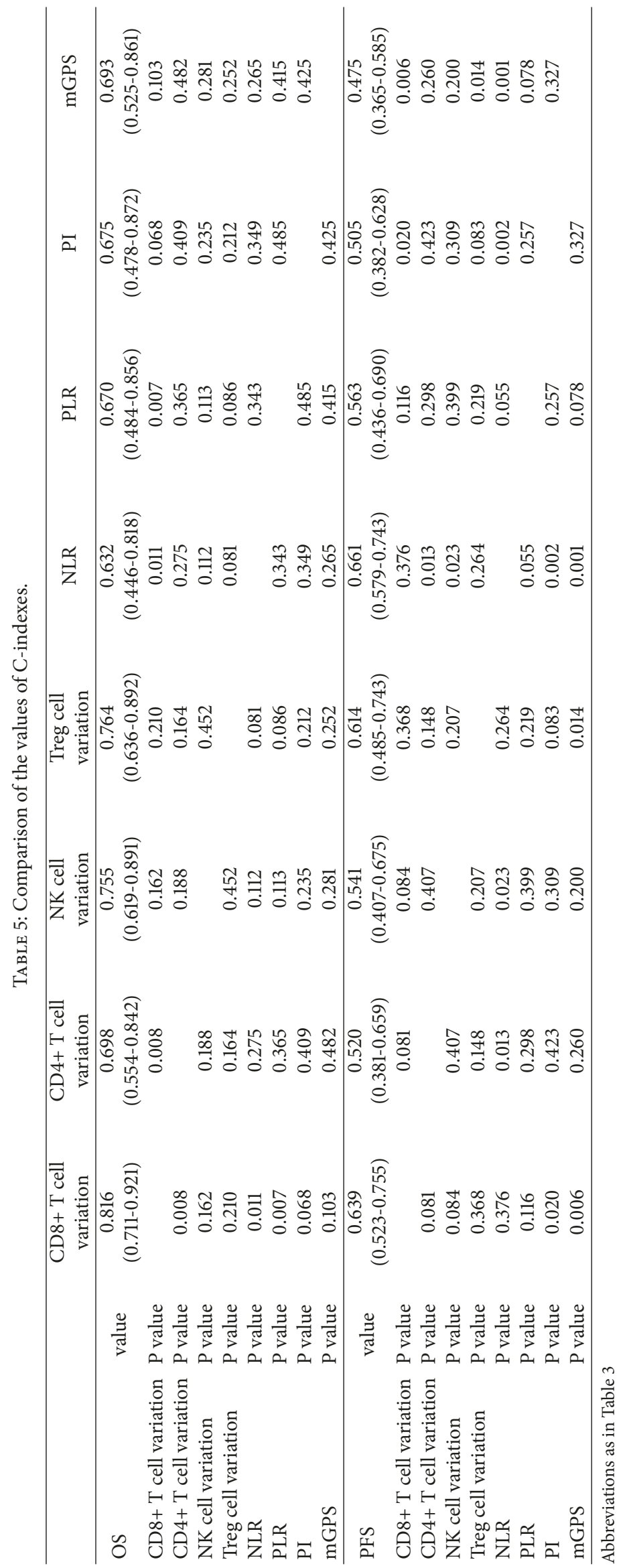


for Treg cell, IL-6, and IL10 after IRE. Other circulating cytokines, including TNF and IFN, were also evaluated. In terms of IFN, IFN- $\gamma$ plays the most important role in its immunostimulatory and immunomodulatory effects, compared with the ability to inhibit viral replication directly, which is the main function of IFN- $\alpha[23,24]$ or IFN- $\beta$ [25]. Therefore, IFN- $\gamma$ and TNF were analyzed while they both failed to show obvious alteration. In addition, the alteration of $\mathrm{CD}^{+} \mathrm{T}$ cell between D3 and D7 was identified as prognostic factor for OS and PFS and first showed both a convenient and effective prognostic value in patients with LAPC after IRE. When compared with the traditional inflammationbased scores, the alteration of $\mathrm{CD}^{+} \mathrm{T}$ cell exhibited a better predictive value for both OS and PFS.

For LAPC patients after ablation therapy, several studies have revealed the changes of individual counts of $T$ cell and subset ratios [26-28]. Alessandro $\mathrm{G}$ et al. compared the concentration of $\mathrm{CD}^{+}$and $\mathrm{CD} 8^{+} \mathrm{T}$ cell before and after radiofrequency ablation (RFA) and revealed an increase of above-mentioned $\mathrm{T}$ cells from the third day after treatment [26]. In the study conducted by Ketevan $M$ et al., a more significant decrease in the expression of $\mathrm{CD}_{4}^{+} \mathrm{CD} 39^{+} \mathrm{T}$ cell was observed after RFA, compared with operation [27]. In animal study, IRE therapy, which induced an increase tumor infiltration of $\mathrm{CD}^{+}$cells, was reported to be more effective in immunocompetent tumor than in immunocompromised tumors [28]. Furthermore, Martin et al. reported that IRE induced an obvious decrease in the absolute number of Treg cell in patients with LAPC [29]. Similar to Martin's study, our study showed a transitory increase followed by a remarkable decrease for Treg cell, along with a steady increase of effective $\mathrm{T}$ cells and humoral immune parameters after IRE. As an inflammation-inducing treatment, IRE not only directly destroys tumor cells, but also results in a release of tumor-associated neoantigens, which may stimulate the cellular and humoral immune of the body. Then, the numbers of immunocytes and production of humoral immune parameters will increase due to the potentiation of cellular and humoral immune. Moreover, it was shown that heatshock proteins released from the destroyed tumor cells had an adjuvant effect and acted as an alarm for antitumor $\mathrm{T}$ cell-mediated immunity [30]. Therefore, IRE may be a mean of significant effort to overcome the immunosuppressive "cold" tumor microenvironment in LAPC and a potential treatment window of opportunity for immune-check-point therapy was suggested by increasing the effector $\mathrm{T}$ cells and decreasing immunosuppressive Treg cells. In addition, for these patients, prior biliary drainage procedure or a hepaticojejunostomy during open procedure was performed. Also, no serious complications, such as abdominal infection and pancreatic fistula, were observed in all patients after IRE therapy. Therefore, the influence of hyperbilirubinemia or infection after IRE on the alteration of immune cells was minimized. Although detained changes of immune cells had been described in patients with LAPC after IRE, the clinical performance of these changes in survival prediction was still unclear, thereby limiting their value.

In the next step of the present study, we evaluated the prognostic factors for OS and PFS and showed that elevation of $\mathrm{CD}^{+} \mathrm{T}$ cell was associated with favourable OS and PFS in LAPC patients after IRE. This can be explained by a stimulated host immune response which might limit the progression and invasion of tumor, and therefore, better survival was achieved. This can be proved by previous studies in which strong relationships were observed between immune toxicity and metastasis [31]. Metastases were more frequently observed in patients with lower density of immune effector cells [32], which was in accordance with our results. Furthermore, the predictive power of the alteration of several immune cells and inflammation-based indexes were compared in this study. It was demonstrated that the alteration of $\mathrm{CD}^{+} \mathrm{T}$ cell was superior to other indexes. In addition, as a robust and economic method, the alteration of $\mathrm{CD}^{+} \mathrm{T}$ cell can be obtained from peripheral blood sample fast and easily and can be used widely in clinical practice. Although there was a correlation between immune cells and inflammationbased indexes [27], the alteration of CD8+ T cell could still provide additional prognostic value in patients with the same levels of inflammation-associated situation. Maybe they can be considered as complements for predicting the prognosis of LAPC patients after IRE. However, a slightly lower value of AUC for CD8+ T cell in PFS prediction suggested that, compared with PFS, maybe OS was affected more greatly by the changes of immune system. Different from tumorinfiltrating $\mathrm{CD}^{+} \mathrm{T}$ cells, which were shown to play more important role in determine local progression, compared with prognosis [33,34]. LAPC is a systemic disease other a local disease. In the present study, peripheral blood samples were collected before and after IRE treatment and were analyzed by flow cytometry for $\mathrm{CD}^{+} \mathrm{T}$ cells. Therefore, it was thought that the human immune system played a more important role in long-term survival than in local control. Similar to the present study, in the study conducted by Chen et al. [35], it was shown that alteration of $\mathrm{CD} 8^{+}$ $\mathrm{T}$ cells was the only independent prognostic factor for OS, other than PFS. This may partly explain the different impact on survival from the alteration of $\mathrm{CD}^{+} \mathrm{T}$ cells. However, this difference needed to be further explored by further studies.

The comparison of AUC and C-indexes of the alteration of immune cells or inflammation-based indexes was conducted. Although the alteration of $\mathrm{CD}^{+} \mathrm{T}$ cell exhibited the most significant effects in predicting survival, statistical significance was not observed for the differences between alteration of $\mathrm{CD}^{+} \mathrm{T}$ cell and some other indexes, implying that the need of improvement in predicting short-term survival with the changes of immune cells. Maybe the magnitude of effector $\mathrm{T}$ cell was more positively associated with longterm survival than short-term survival [36].

As the first study to compare the changes of immunocytes and to explore the prognostic power of these changes in patients with LAPC after IRE, our study was limited by the small size and retrospective nature. The immunocytes measured in this study did not represent all the components in the microenvironment of LAPC after IRE therapy. Moreover, maybe it is necessary to analyze the immune parameters withdrawn at the moment of progression. A longer followup period is also needed for the comparisons of effects of 
immunocytes on survival in LAPC patients and an external validation is also needed.

\section{Conclusions}

The present study showed the first picture of immunomodulatory of IRE in patients with LAPC. Alteration of CD8 ${ }^{+}$ $T$ cell was established as prognostic factor for OS and PFS and showed better prognostic value for survival prediction in LAPC patients after IRE therapy. The changes of $\mathrm{CD}^{+}$ T between D3 and D7 after IRE could be used as a monitor factor of IRE treatment and a prognostic indicator of survival in LAPC patients after IRE therapy.

\section{Data Availability}

The datasets from SYSUCC dataset are available from the corresponding author upon reasonable request.

\section{Conflicts of Interest}

All authors declare that they have no conflicts of interest.

\section{Authors' Contributions}

Chaobin He, Jun Wang, Shuxin Sun, and Yu Zhang collected the dataset; Chaobin He, Jun Wang, and Shuxin Sun performed the statistical analyses and wrote the manuscript; Shengping Li designed the study. Chaobin He, Jun Wang, and Shuxin Sun contributed equally to this work.

\section{Acknowledgments}

This work was supported by grants from the National Natural Science Funds (no. 81672390) and the National Key Research and Development Plan (no. 2017YFC0910002).

\section{References}

[1] F. Bray, J. Ferlay, and I. Soerjomataram, "Global cancer statistics 2018: GLOBOCAN estimates of incidence and mortality worldwide for 36 cancers in 185 countries," CA: A Cancer Journal for Clinicians, vol. 68, no. 6, pp. 394-424, 2018.

[2] W. Chen, R. Zheng, P. D. Baade et al., "Cancer statistics in China, 2015," CA: A Cancer Journal for Clinicians, vol. 66, no. 2, pp. 115$132,2016$.

[3] M. Bockhorn, F. G. Uzunoglu, M. Adham et al., "Borderline resectable pancreatic cancer: a consensus statement by the International Study Group of Pancreatic Surgery (ISGPS)," Surgery, vol. 155, no. 6, pp. 977-988, 2014.

[4] E. P. Balaban, P. B. Mangu, A. A. Khorana et al., "Locally advanced, unresectable pancreatic cancer: American society of clinical oncology clinical practice guideline," Journal of Clinical Oncology, vol. 34, no. 22, pp. 2654-2668, 2016.

[5] R. A. Abrams, A. M. Lowy, E. M. O’Reilly, R. A. Wolff, V. J. Picozzi, and P. W. T. Pisters, "Combined modality treatment of resectable and borderline resectable pancreas cancer: expert consensus statement," Annals of Surgical Oncology, vol. 16, no. 7, pp. 1751-1756, 2009.
[6] V. Moutardier, V. Magnin, O. Turrini et al., "Assessment of pathologic response after preoperative chemoradiotherapy and surgery in pancreatic adenocarcinoma," International Journal of Radiation Oncology • Biology • Physics, vol. 60, no. 2, pp. 437443, 2004.

[7] M. Suker, B. R. Beumer, E. Sadot et al., "FOLFIRINOX for locally advanced pancreatic cancer: a systematic review and patient-level meta-analysis," The Lancet Oncology, vol. 17, no. 6, pp. 801-810, 2016.

[8] T. Conroy, P. Hammel, M. Hebbar et al., "FOLFIRINOX or gemcitabine as adjuvant therapy for pancreatic cancer," The New England Journal of Medicine, vol. 379, no. 25, pp. 2395-2406, 2018.

[9] H. Kang, J. H. Jo, H. S. Lee et al., "Comparison of efficacy and safety between standard-dose and modified-dose FOLFIRINOX as a first-line treatment of pancreatic cancer," World Journal of Gastrointestinal Oncology, vol. 10, no. 11, pp. 421-430, 2018.

[10] M. G. Keane, K. Bramis, S. P. Pereira, and G. K. Fusai, "Systematic review of novel ablative methods in locally advanced pancreatic cancer," World Journal of Gastroenterology, vol. 20, no. 9, pp. 2267-2278, 2014.

[11] R. C. Martin, D. Kwon, S. Chalikonda et al., "Treatment of 200 locally advanced (stage III) pancreatic adenocarcinoma patients with irreversible electroporation: safety and efficacy," Annals of Surgery, vol. 262, no. 3, pp. 486-494, 2015.

[12] M. J. Weiss and C. L. Wolfgang, "Irreversible electroporation," Advances in Surgery, vol. 48, no. 1, pp. 253-258, 2014.

[13] J. Lavee, G. Onik, P. Mikus, and B. Rubinsky, "A novel nonthermal energy source for surgical epicardial atrial ablation: irreversible electroporation," The Heart Surgery Forum, vol. 10, no. 2, pp. E162-E167, 2007.

[14] S. B. White, Z. Zhang, J. Chen, V. R. Gogineni, and A. C. Larson, "Early immunologic response of irreversible electroporation versus cryoablation in a rodent model of pancreatic cancer," Journal of Vascular and Interventional Radiology, vol. 29, no. 12, pp. 1764-1769, 2018.

[15] X. Li, K. Xu, W. Li et al., "Immunologic response to tumor ablation with irreversible electroporation," PLOS ONE, vol. 7, no. 11, Article ID e48749, 2012.

[16] M. S. Lawrence, P. Stojanov, P. Polak et al., "Mutational heterogeneity in cancer and the search for new cancer-associated genes," Nature, vol. 499, no. 7457, pp. 214-218, 2013.

[17] D. R. Byrd, C. C. Compton, A. G. Fritz, F. L. Greene, and A. Trotti, Eds., AJCC Cancer Staging Manual, Springer, New York, NY, USA, 7th edition, 2010.

[18] M. P. Callery, K. J. Chang, E. K. Fishman, M. S. Talamonti, L. William Traverso, and D. C. Linehan, "Pretreatment assessment of resectable and borderline resectable pancreatic cancer: expert consensus statement," Annals of Surgical Oncology, vol. 16, no. 7, pp. 1727-1733, 2009.

[19] C. He, X. Lin, and S. Lu, "Inflammation scores predict the survival of patients with hepatocellular carcinoma who were treated with transarterial chemoembolization and recombinant human type-5 adenovirus H101," PLoS ONE, vol. 12, no. 3, Article ID e0174769, 2017.

[20] J. Huang, J. Huang, Z. Duan et al., “Th2 predominance and CD8+ memory $\mathrm{T}$ cell depletion in patients with severe acute respiratory syndrome," Microbes and Infection, vol. 7, no. 3, pp. 427-436, 2005.

[21] C. He, Y. Mao, J. Wang, F. Duan, X. Lin, and S. Li, "Nomograms predict long-term survival for patients with periampullary 
adenocarcinoma after pancreatoduodenectomy," BMC Cancer, vol. 18, no. 1, p. 327, 2018.

[22] C. He, Y. Mao, J. Wang, X. Huang, X. Lin, and S. Li, "Surgical management of periampullary adenocarcinoma: defining an optimal prognostic lymph node stratification schema," Journal of Cancer, vol. 9, no. 9, pp. 1667-1679, 2018.

[23] E. A. Caine, S. M. Scheaffer, N. Arora et al., "Interferon lambda protects the female reproductive tract against Zika virus infection," Nature Communications, vol. 10, no. 1, p. 280, 2019.

[24] L. Zhou, J. Ren, S. Cheng et al., "A functional variant in ubiquitin conjugating enzyme E2 L3 contributes to hepatitis B virus infection and maintains covalently closed circular DNA stability by inducing degradation of apolipoprotein B mRNA editing enzyme catalytic subunit 3A," Hepatology, vol. 69, no. 5, pp. 1885-1902, 2019.

[25] R. Kuo, C. Chen, R. Y. Wang et al., "Role of enteroviral RNAdependent RNA polymerase in regulation of MDA5-mediated beta interferon activation," Journal of Virology, vol. 93, no. 10, 2019.

[26] A. Giardino, G. Innamorati, S. Ugel et al., "Immunomodulation after radiofrequency ablation of locally advanced pancreatic cancer by monitoring the immune response in 10 patients," Pancreatology, vol. 17, no. 6, pp. 962-966, 2017.

[27] K. Mazmishvili, K. Jayant, N. Janikashvili et al., "Study to evaluate the immunomodulatory effects of radiofrequency ablation compared to surgical resection for liver cancer," Journal of Cancer, vol. 9, no. 17, pp. 3187-3195, 2018.

[28] R. E. Neal II, J. H. Rossmeisl Jr., J. L. Robertson et al., "Improved local and systemic anti-tumor efficacy for irreversible electroporation in immunocompetent versus immunodeficient mice," PLoS ONE, vol. 8, no. 5, Article ID e64559, 2013.

[29] H. Pandit, Y. K. Hong, Y. Li et al., "Evaluating the regulatory immunomodulation effect of irreversible electroporation (IRE) in pancreatic adenocarcinoma," Annals of Surgical Oncology, vol. 26, no. 3, pp. 800-806, 2019.

[30] S. P. Haen, C. Gouttefangeas, D. Schmidt et al., "Elevated serum levels of heat shock protein 70 can be detected after radiofrequency ablation," Cell Stress and Chaperones, vol. 16, no. 5, pp. 495-504, 2011.

[31] B. Mlecnik, G. Bindea, A. Kirilovsky et al., "The tumor microenvironment and Immunoscore are critical determinants of dissemination to distant metastasis," Science Translational Medicine, vol. 8, no. 327, Article ID 327ra26, 2016.

[32] Y. Wang, H. Lin, M. Huang et al., "The Immunoscore system predicts prognosis after liver metastasectomy in colorectal cancer liver metastases," Cancer Immunology, Immunotherapy, vol. 67, no. 3, pp. 435-444, 2018.

[33] S. Shimizu, H. Hiratsuka, K. Koike et al., “ Tumor-infiltrating CD8 + T-cell density is an independent prognostic marker for oral squamous cell carcinoma ," Cancer Medicine, vol. 8, no. 1, pp. 80-93, 2019.

[34] X. Xu, Y. Tan, Y. Qian et al., "Clinicopathologic and prognostic significance of tumor-infiltrating CD8+ T cells in patients with hepatocellular carcinoma," Medicine, vol. 98, no. 2, p. e13923, 2019.

[35] X. Chen, W. Zhang, D. Qian et al., "ChemoradiotherapyInduced CD4+ and CD8+ T-cell alterations to predict patient outcomes in esophageal squamous cell carcinoma," Frontiers in Oncology, vol. 9, p. 73, 2019.

[36] S. C. Katz, V. Pillarisetty, Z. M. Bamboat et al., "T cell infiltrate predicts long-term survival following resection of colorectal cancer liver metastases," Annals of Surgical Oncology, vol. 16, no. 9, pp. 2524-2530, 2009. 


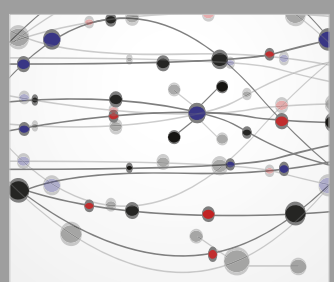

The Scientific World Journal
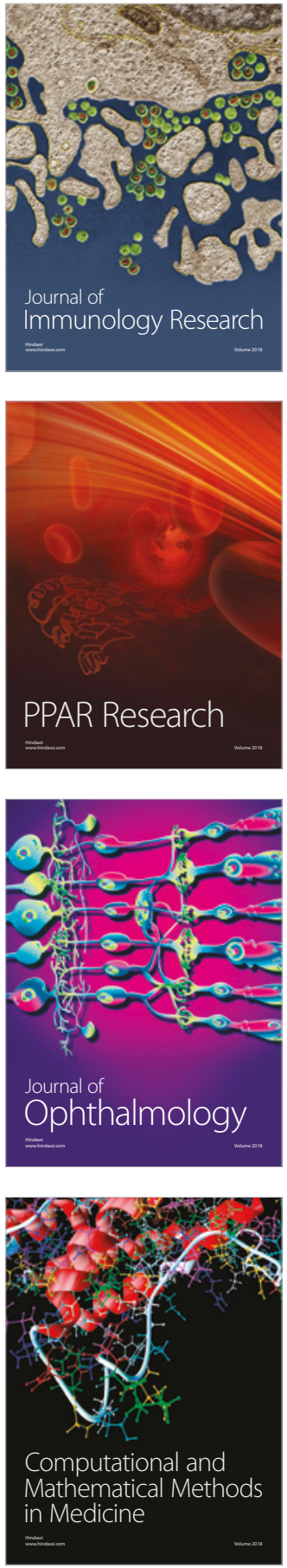

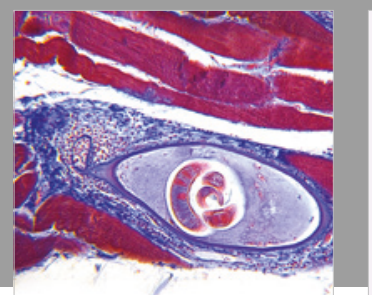

Gastroenterology Research and Practice

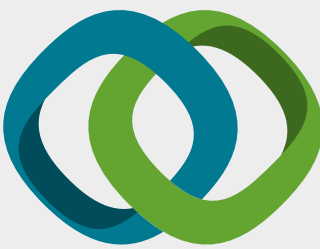

\section{Hindawi}

Submit your manuscripts at

www.hindawi.com
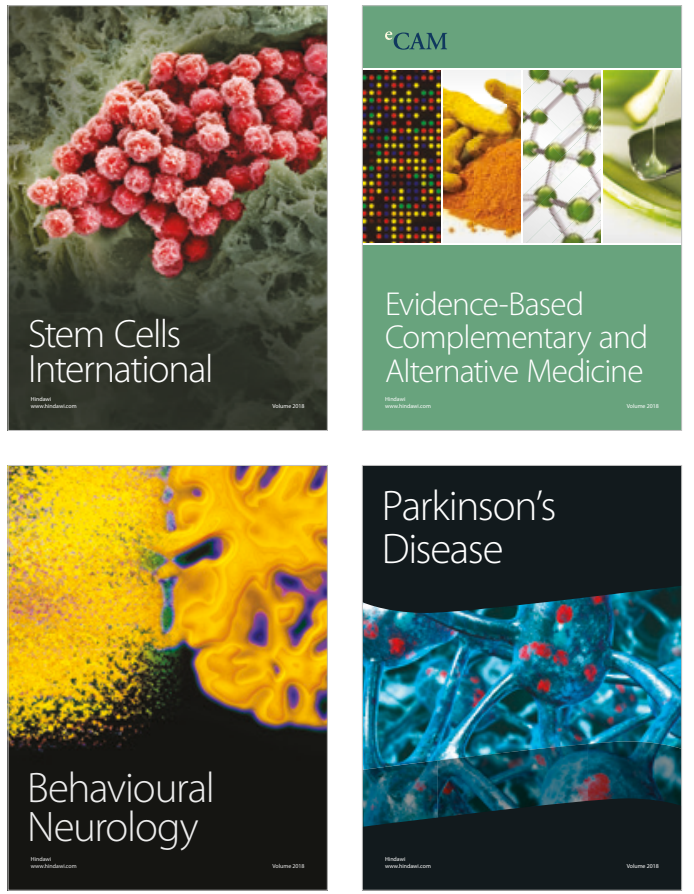

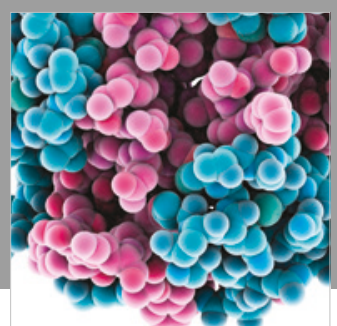

ournal of

Diabetes Research

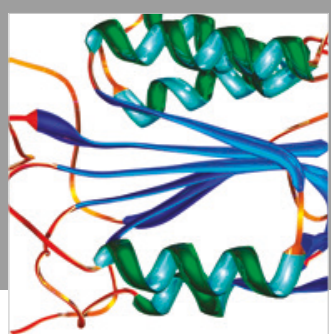

Disease Markers
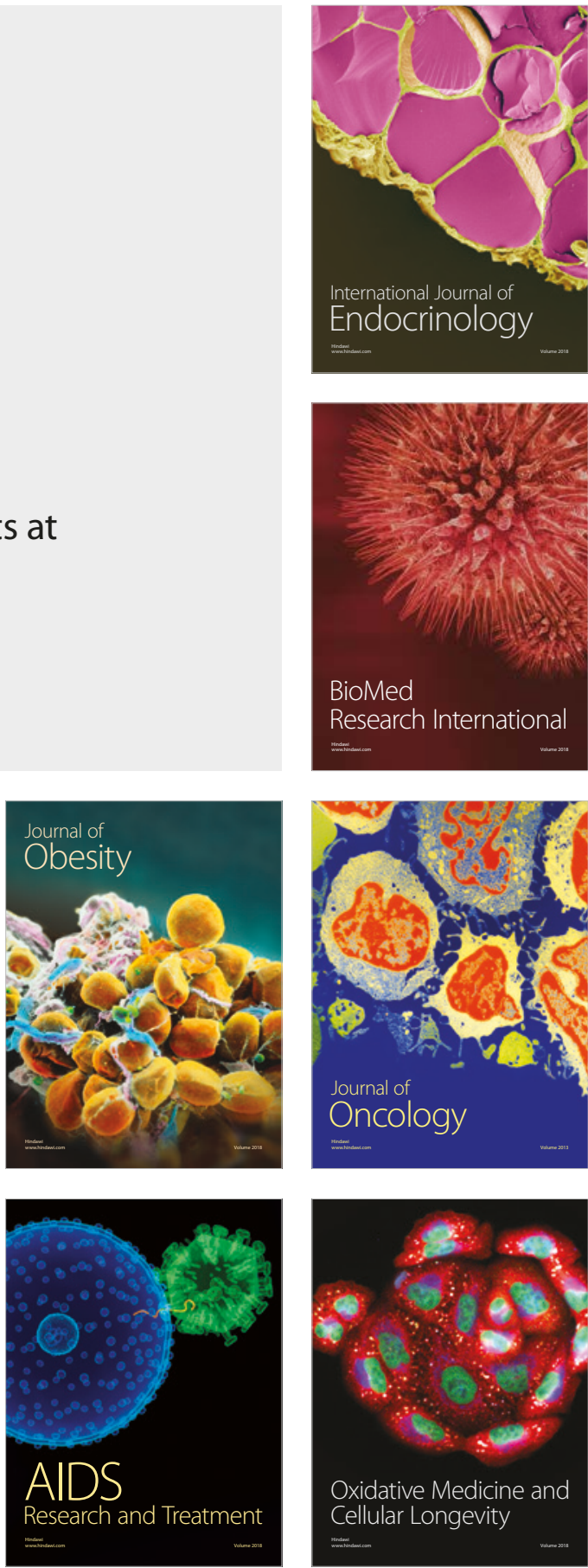